Viewpoints and Letters to the Editor are published in Hort- comments on matters of concern to horticulturists. These are Science to provide members of the American Society for Hor- not statements of official Society policy nor do they necessarticultural Science an opportunity to share their experiences and ily reflect the views of a majority of the Society's members.

\title{
Teaching Philosophy and Techniques
}

\author{
Marlin N. Rogers ${ }^{1}$ \\ Department of Horticulture, University of Missouri- Columbia, Columbia, MO 65211
}

\begin{abstract}
My philosophy and techniques of teaching have developed gradually over almost 40 years of college instruction, from which I have derived a great deal of pleasure and personal satisfaction. I looked forward eagerly to each day in the classroom, and have worked with many dedicated and talented students. Listed below are some of the educational principles that I consider important, and the techniques that I used to implement them.

1) Always have high performance expectations for your students. Mine respected me for that belief; and, in most cases, rose to the challenge. I wanted them to feel that they got their money's worth from my course. Sometimes they complained at the time, but now I am sure they appreciate my concern.

2) Build from the ground up. I always tried to be sure they understood the. fundamentals before going on to more complex subjects. Students quickly lose interest and incentive if they get lost in the early stages of learning.
\end{abstract}

During my first years of teaching, I assigned a major outside class project with a completion date at the end of the semester. This resulted in much trauma and loss of sleep during the final week of the semester, and often a rather poorly done project. I finally modified my procedure by establishing a series of deadlines, with the first early in the semester. This change forced the students to begin working on the project early enough to do it justice, and also let me determine whether or not they understood the fundamentals before starting on the more involved aspects. If they did not understand the early steps, I could get them back on track before they tried to complete the later ones. Thus, much student desperation, despair, and loss of self-esteem were prevented, and the overall educational climate was greatly improved.

3) When teaching, try to inculcate a basic understanding of fundamental principles, from which your students can then derive applications by reason, rather than memory.

The response of plants to their environment is based on the fundamental principles of chemistry, physics, mathematics, genetics, and plant physiology. Students may have learned something about these in their basic science courses, but often are not familiar with the organisms used as examples.

Received for publication 8 Sept. 1989.

${ }^{1}$ Professor Emeritus.

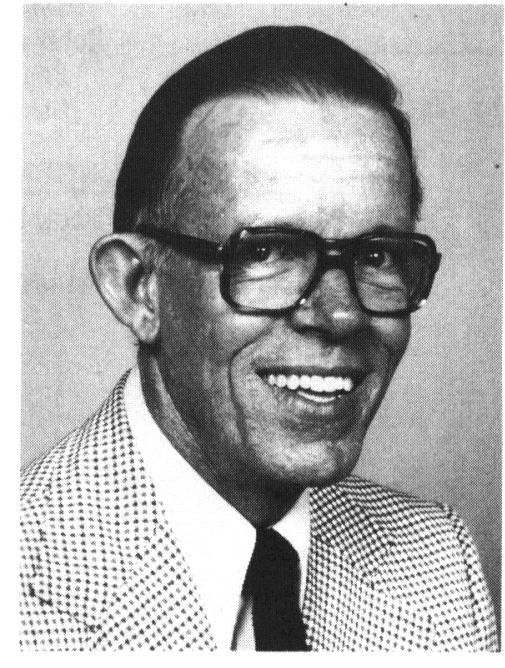

A review of these basic facts, as illustrated by the responses of some of the horticultural plants with which they are familiar, finally begins to generate a real understanding of the topic. Hearing about the effects of photoperiod on Xanthium penlsylvanicum Wallr. in a basic botany course may not mean much to a student, but talking about the same response in relation to poinsettias or chysanthemums, which are familiar plants to them, is more understandable.

One fall I purposely had my students grow a few pot mums on each bench in our teaching greenhouse. A street lamp was outside one corner of the structure. Six or 8 weeks after daylengths were supposed to be short enough for flowering, the students whose plants were closest to the lamp began to wonder why their plants were still green, while their peers at the other end of the greenhouse had plants beginning to show color. My second lecture on photoperiodism that semester (the first had been earlier in the classroom when we discussed mum culture) did not have to be very long, but I'm quite sure all the students got a new understanding of photoperiodism that day-one they never forgot.

4) Emphasize the importance of the practical applications of the basic scientific principles you teach. Theoretical information about plant growth is of little value to a horticulturist until the knowledge can be applied in the day-to-day plant production problems, whether it be in business, research, or hobby pursuits.

5) Have as a goal for students an understanding of the common threads running through the production and culture of all kinds of plants, so that they can approach the culture of new or unfamiliar species with confidence.

With the above principles in mind, many of the more important flower crops were grown in the greenhouse during the greenhouse crops production laboratories. I provided all the inputs needed as well as advice and counsel. Then I stood back. Each small group of students was completely responsible for producing its crop (except for weekend watering, which the staff provided).

By growing many different kinds of plants, the students were able to observe the commonalities involved-the favorable responses of all plants to good cultural practices, regular watering, proper fertilization, carefully planned pest control regimes, etc.

6) When discussing the culture of individual crops, try to emphasize the responses that are unique. Generally, these characteristics have to be memorized because they can not be derived from application of general principles.

While my students were watching for cultural similarities in the crops they were growing, they also began to experience the unique and diverse responses of the various crops. One student grew the best Exacums in the entire class, but the absolute worst poinsettias and pot mums. He was a negligent waterer, and only irrigated his plants once a week on laboratory day (I also watered once on Sunday). Others, who watered more conscientiously, had excellent poinsettias and pot mums, but some lost their Exacums to Botrytis rot as a result of daily watering.

We had the students critique their own and their peers' production results frequently, and to diagnose problems that had been discussed earlier in the classroom, but which they were now seeing in the real world. In this way they were approaching learning in a different way, which reinforced the earlier classroom discussions.

One key to success in this type of teaching is critical. You must not rescue your students from their mistakes! If you walk through the greenhouse on Wednesday, and some of the plants are dry to the point of death, you must not water them! Students have to learn about the consequences of negligence if they are to become competent horticulturists.

7) When you are with your students in class, remember that nothing is more important than their comprehension of the subject matter being considered. It is not the teacher's function to entertain them or pro- 
vide a relaxing interlude until the next important activity in their day.

Good teaching is hard work and requires the total concentration of both teacher and student. As the instructor, it is your responsibility to present difficult material so that it can be comprehended and understood by every student in the class.

When the organization of a given presentation was at all complicated, I tried to help the students to follow it by the use of an outline of the lecture on the overhead projector. This practice was beneficial to me as well, by keeping me on track, and preventing wandering and distraction.

8) Make the subject more interesting and understandable by extensive use of visual aids that illustrate the principles being discussed, and/or some of the applications of them. I have been fortunate to be able to travel to many different parts of the world, and the ideas shown in some of the slides I shared with my students opened up new vistas and aroused much interest among them. Showing how others approach the problems presented by our profession almost always gets students interested and enthusiastic about the subject. Most of us are interested in far-away places, and the chance to see how others do things is always stimulating.

9) It is critical that you present current and updated information. If the teacher is not upto-date in the subject matter being presented, why is he or she being paid money to disseminate knowledge?

From the very beginning of my teaching career, I always collected and filed scientific reprints and copies of trade paper articles, and reviewed these each year while preparing my lecture material. In this way, both teacher and students were able to keep abreast of current research and the new developments in crop production being generated around the world.

10) Because truth is not always easy to discern, try to avoid being an opinionated teacher and do not approach problems from an emotional point of view.

When presenting distinctly controversial topics, I always tried to present the arguments on both sides of the question, and encouraged the students to reach their own conclusions, based on their critical and objective thinking rather than on their emotions. This helped them arrive at a more balanced view of the problem than they might have assumed initially. I tried to get them to think positively about the problem, and look upon it as an opportunity to do something in a better way or improve the situation in the future.
For example, florists may shortly be forced legally to severely reduce greenhouse effluents containing fertilizers and pesticides, which could contaminate underground water supplies. However, rather than wailing about it, maybe we need to reassess our watering, fertilization, and pest control techniques to overcome the problem. If we use ebb and flow watering systems with recirculating fertilizer solutions and low-volume pesticide application methods that greatly reduce the total quantities of pesticides applied, we have an opportunity to grow better, higher-quality plants at possibly lower labor and materials costs.

11) Be helpful and willing to spend extra time with individual students who, for one reason or another, are having difficulty with the subject matter being taught.

I always had an open-door policy, and took the time to assist my students having problems understanding material presented in class. These sessions often provided me feedback, and alerted me to poor presentations that needed to be improved, or to the fact that these particular students were in need of additional background information before they could really understand the topic.

12) Be adaptable to the needs of your audience. I found out early that about half of my students consistently did better on discussion questions on my exams than on objective-type questions, and the other half were exactly the opposite. To be as fair as possible to everyone, I always split my examinations about equally between the two types of questions. Since one can determine the student's understanding of the subject by the use of either type of question, why not be adaptable?

Very often I received answers to exam questions that I had not considered at all when writing the question. In those cases, when the question could be interpreted logically in the way the students had done, I gave them full credit for their answers. There is often more than one logical way to interpret a given statement. Students may not be clairvoyant, but can be just as logical as you are!

13) Make your examination days as fruitful in learning as the days on which you lecture or conduct laboratories. I tried to make my exam questions interesting and challenging questions that really made the students think and use their reasoning ability to answer. When the papers were returned, I always used a little class time to go over the questions in some detail. Much real learning occurred during these sessions, because I had the students' complete attention.

14) Help your students integrate the new information learned in your course with their previous knowledge.

To help my students integrate all their knowledge about greenhouse crops, I had them complete, at the end of the course, a year-long crop rotation plan for a sizeable commercial greenhouse. They had to provide for salable crops on a weekly or biweekly basis throughout the year, with significantly larger quantities of production at the major flower holidays. I provided them with estimated costs of propagation material, approximate selling prices for the items to be produced, estimated dumping percentages and special selling costs, and. estimated overhead costs to cover all other items of production expense.

With this basic information, they were expected to prepare crop rotation plans, calculate approximate costs of production and relative potential profitability of the different crops included on their plan, and, at the end, an estimate of their total profit or loss for the year's operations. Their goal was to achieve a high percentage of use of the bench space available and maximum net profit, while at the same time producing items that would have a reasonably good chance of being grown and sold in our area under current conditions.

For years, all of the calculations had to be carried out manually, and results were presented in terms of worksheets and graphical summaries. The last few years I taught the course, we were able to do all the calculations using spreadsheet programs on our computers. This added a new dimension of learning, since some of the students had not had a basic computing course before enrolling in mine. However, by spending some extra time with this group, we were able to teach them enough about computing that they ended up with results equal in quality to those who had had some previous computer experience.

Good teaching is not made up of occasional heroic and glorious presentations worthy of Olympic medals! It consists of the daily performance of a multitude of little acts, each of which would be more suitably recognized with something like the Good Conduct medal.

Conscientious and caring teachers bless the lives of hundreds of young people, and are key contributors to the economic future of our land. May the value of their work be recognized and suitably rewarded by our American society, whose wellbeing is so dependent upon them! 